\title{
Synergistic Regulation of Human Megakaryocyte Development
}

Michael W. Long, Raymond J. Hutchinson, Linda L. Gragowski, Connie H. Heffner, and Stephen G. Emerson*

Hematology/Oncology Divisions, Departments of Pediatrics and Internal Medicine, ${ }^{*}$ University of Michigan, Ann Arbor, Michigan 48109

\begin{abstract}
Little information exists concerning differing levels of regulation occurring during human megakaryocyte development. We hypothesize that megakaryocytic proliferation and maturation is controlled by two, synergistic regulatory factors. One, megakaryocyte colony-stimulating activity, is an obligate requirement for colony formation and drives the development of relatively immature cells. Megakaryocyte colony-stimulating activity is a functional component of the human recombinant proteins, interleukin 3 or GM-CSF. Human recombinant growth factors, interleukin 1, interleukin 6, or crythropoietin, do not effect megakaryocyte development either alone or in combination with interleukin 3 . Full maturation requires a second synergistic activity which increases megakaryocyte number, size, and cytoplasmic and antigenic content. In culture, this synergistic regulator augments maturation by increasing the number of colonies, colony cellularity, and size. In suspension cultures, this cofactor increases megakaryocyte cytoplasmic and antigenic content, and shifts the morphological distribution from immature to mature megakaryocytes. Finally, this activity also increases the number of antigen positive megakaryocytes, either by stimulating proliferation or conversion of antigen-negative to antigen-positive cells. Comparative studies of megakaryocytic regulation suggests that this in vitro regulator mimicks some of the known effects of thrombopoietin in vivo.
\end{abstract}

\section{Introduction}

The existence of multiple regulators that act to coordinate hematopoietic differentiation is established for erythrocytic and granulocytic progenitor cells (1-4). In the megakaryocytic lineage, studies on the sera of patients with platelet/megakaryocyte dyscrasias suggest a similar pluralistic control of megakaryocyte development $(5,6)$. In vitro studies show that megakaryocyte colony formation requires an activity that regulates progenitor cell proliferation $(6,7)$. We hypothesized that the regulation of human megakaryocyte colony development may also require additional activities that augment megakaryocyte colony development presumably by affecting differentiation (8-11).

Various sources of human megakaryocyte colony-stimulating activities (Mk-CSA) ${ }^{1}$ exist (12-16). Among these are Received for publication 2 September 1986 and in revised form 22 June
1988 .

1. Abbreviations used in this paper: $\mathrm{BICM}$, human bladder carcinoma (5637)-conditioned media; BPA, burst-promoting activity; CM, con-

J. Clin. Invest.

(C) The American Society for Clinical Investigation, Inc.

$0021-9738 / 88 / 11 / 1779 / 08 \quad \$ 2.00$

Volume 82, November 1988, 1779-1786 conditioned media from phytohemagglutinin-stimulated peripheral blood leukocytes (PHA-LCM) $(12,13)$, or plasma/ sera obtained from patients with aplastic anemia (14), amegakaryocytic thrombocytopenia (15), or rebound phases of chemotherapy (16). However, scarcity, problems with sample complexity, and patient/donor variability make these difficult sources for purification and/or in vitro characterization of megakaryocyte regulators.

We report that optimal in vitro human megakaryocyte colony development requires two regulatory activities. Mk-CSA, a functional activity of interleukin 3 (IL-3) or granulocyte/ macrophage colony-stimulating factor (GM-CSF), is obligate for colony formation and alone stimulates limited megakaryocyte development. A second auxiliary activity, which lacks the solitary ability to stimulate megakaryocyte colonies, acts synergistically with Mk-CSA to augment megakaryocyte colony development. Moreover, this activity regulates megakaryocytic size and the expression of megakaryocytic antigens.

\section{Methods}

\section{Preparation of bone marrow cells}

After we received informed consent, bone marrow aspirates were obtained from the posterior iliac crest of normal volunteers $(n=41$, age 18-35 yr, 24 males, 17 females). Volunteers were reportedly free of pharmaceuticals for 3 wk before the aspiration.

Bone marrow cells were aspirated into syringes containing $100 \mathrm{U}$ of preservative-free sodium heparin (University of Michigan) in normal saline. In order to prevent peripheral blood dilution, aspirate volumes were kept at $0.5-0.8 \mathrm{ml}$. Aspirates were immediately mixed with $100 \mu \mathrm{l}$ of McCoy's 5A media (see below) containing 100-125 U DNase I (Sigma Chemical Co., St. Louis, MO). The addition of DNase to the samples improved the recovery of cells after density separation. The aspirate was diluted with McCoy's 5A media (Gibco, Grand Island, New York), layered over ficoll/diatrizoate (density $=1.077 \mathrm{~g} \mathrm{~cm}^{-3}$, Histopaque, Sigma Chemical Co.), and spun for $20 \mathrm{~min}$ at $400 \mathrm{~g}$ (mononuclear cell recovery $=7.9 \pm 4.6 \times 10^{7}$ cells, $n=17$ ). The interface cells were collected, washed twice, and subjected to two rounds of plastic adherence. For adherence, low-density marrow cells were incubated $\left(3 \times 10^{5} / \mathrm{ml}\right)$ in McCoy's 5 A containing $5 \%$ FCS for $1 \mathrm{~h}$ at $37^{\circ} \mathrm{C}$, after which the suspension cells were removed and subjected to a second adherence step. The resultant nonadherent, low-density cells (NALD; $\leq 0.1 \%$ macrophages) were used as target cells in all experiments.

\section{Hematopoietic progenitor cell assays}

Progenitor cells were cultured in methylcellulose-based assays as described by Messner et al. (12), and in a modification of a previously described agar system $(10,11)$. Methylcellulose assays were used to

ditioned media; CSA, colony-stimulating activities; CSF, colony-stimulating factor; GM, granulocyte macrophage; HEL, human erythroleukemia; HLuCM, human lung-conditioned media; NALD, nonadherent low-density bone marrow cells; rh, recombinant human; SPA, staphylococcal protein A; SPRIA, solid-phase radioimmunoassay; TNC, total nucleated cells. 
examine the effects of erythropoietin on megakaryocyte colony formation.

Methylcellulose cultures. Bone marrow NALD cells $\left(1.0 \times 10^{5} / \mathrm{ml}\right)$ were suspended in Iscove's modified Dulbecco's MEM (IMDM, Gibco) containing $5 \times 10^{-5} \mathrm{M}$ 2-mercaptoethanol (Sigma Chemical Co.) and made semisolid by the addition of 0.9-1.0\% methylcellulose (Methylcel-MC, 4,000 mPa.s, Fluka AG, Buchs, Switzerland). Routinely, human plasma, obtained from normal donors, was added at a concentration of $30 \%$. In addition, these cultures contained $10 \%$ PHA-LCM. Recombinant erythropoietin (TC Epo, Amgen, Thousand Oaks, CA) was added at $2 \mathrm{U} / \mathrm{ml}$. Cultures were incubated in $35-\mathrm{mm}$ petri dishes (Lux, Lab-Tech Div., Miles Laboratories, Naperville, IL) for $14-16 \mathrm{~d}$ at $37^{\circ} \mathrm{C}, 100 \%$ humidity, $7 \% \mathrm{CO}_{2}$.

Semisolid agar cultures. These cultures were performed as described for murine cells $(10,11)$, except that $30 \%$ human plasma replaced the FCS and PGE $_{2}$ is omitted. Briefly, NALD cells were cultured in supplemented McCoy's 5A media containing 30\% heparinized human plasma and $\mathbf{0 . 2 5 \%}$ bactoagar (Difco, Detroit, MI). Bone marrow cells were cocultivated with conditioned medium (CM) from a human carcinoma cell line, human lung (HLu) cell lines, or PHALCM (vide infra). Incubation conditions were identical to those for methylcellulose cultures. Unless otherwise stated, all cultures were plated at a limiting cell density of $5 \times 10^{4}$ NALD cells $/ \mathrm{ml}$ (The CFUMk assay, in agar, was linear over a range of $25-100 \times 10^{3}$ cells $/ \mathrm{ml}, r$ $=0.95, r^{2}=0.90, y$-intercept $=-0.3$ ).

Megakaryocyte colonies were identified by their morphologic characteristics as described elsewhere $(12,13,17)$. Morphologic identification of megakaryocyte colonies (as presented in Results) was validated by immunoperoxidase labeling using platelet-megakaryocyte specific antibodies (see below). Individual marrow sample replicate cultures were air dried (18) and stained by immunoperoxidase labeling. These parallel cultures gave equivalent results to those based on morphologic identification for each of the regulatory activities examined. Thus, immunocytochemical identification yielded $10 \pm 5$ CFC$\mathrm{Mk} / 10^{5}$ total nucleated cells (TNC) for Mk-CSA stimulation; $30 \pm 10$ for cocultivation with Mk-CSA and PMA, and $21 \pm 6$ for human bladder carcinoma CM-derived (vide infra) Mk-CSA plus HLuCM (values are mean $\pm \mathrm{SD}$ for three to five replicate cultures/condition/donor; $n$ = 3; for comparison see values presented in Results). It should be noted that both morphologic or immunocytochemical identification were conservative estimates of megakaryocyte colony frequency. In the case of the former, colonies composed of small (immature) megakaryocytes were not detected. In the latter, colonies of nonresponding, immature (antigen negative) cells were missed.

The sample number reported $(n)$ represents the number of individual marrows examined. For each marrow, all experimental conditions were cultured as three to five replicate cultures/condition. Values are expressed as mean $\pm S D$ of the mean replicate culture values averaged over the indicated number $(n)$ of individual marrow samples.

\section{Conditioned media}

Various CM were used as sources of hematopoietic regulatory activities. These are PHA-LCM, a standard source of such regulatory activities $(12,13)$, and media conditioned by three human cell lines: a bladder carcinoma cell line, 5637 (a generous gift of the late Dr. Jorgen Flogh, Sloan Kettering Institute, Rye, NY), and two lung cell lines (A549 and Hel 299 from American Type Tissue Culture Collection, Rockville, MD).

Human PHA-LCM. Human leukocyte-rich plasma was obtained by admixing peripheral blood (buffy coat) with $2 \%$ methylcellulose (1:2) and the leukocyte-rich plasma was collected 30-60 min later. The cells were washed three times with IMDM, and cultured $\left(1 \times 10^{6} / \mathrm{ml}\right)$ in IMDM containing 10\% FCS and 1\% PHA (Wellcome Diagnostics, Greenville, NC). After $7 \mathrm{~d}, \mathrm{CM}$ was harvested by centrifugation, filter sterilized $(0.22 \mu \mathrm{m})$, and stored at $-80^{\circ} \mathrm{C}$ until used.

Human lung conditioned medium ( $H L U C M$ ). Human lung cells were grown in RPMI 1640 and containing 10\% FCS (Hyclone, Ogden, UT) supplemented with $1.0 \%$ essential amino $(50 \times), 0.75 \%$ nonessential amino acids $(100 \times)$ and $0.75 \%$ vitamins $(100 \times)$, vol/vol (all from
Gibco), $7.5 \%$ sodium bicarbonate, and $0.25 \%$ glucose. These cells were grown to confluence, and media were conditioned for $5 \mathrm{~d}$. Supernatant media were decanted, centrifuged, filter sterilized, and stored at $-80^{\circ} \mathrm{C}$.

Human bladder carcinoma (5637)-conditioned media (BlCM). Adherent human bladder carcinoma cells were grown in RPMI 1640 containing $0.75 \%$ (wt/vol) glutamine (100×, Gibco) and $2 \%$ FCS. Cells were cultured in $75-\mathrm{cm}^{2}$ flasks, on Cytodex-3 beads (Pharmacia Fine Chemicals, Piscataway, NJ). Cells were grown to confluence (on beads), and media were conditioned for 3-4 d. After centrifugation, the CM were concentrated fivefold by ultrafiltration (Amicon YM-10 membrane, Amicon Corp., Lexington, MA), filter-sterilized, and stored at $-80^{\circ} \mathrm{C}$.

Optimal concentrations of these $\mathrm{CM}$ were determined by using reciprocal titrations as reported elsewhere (19). These CM were examined over a range of $0.1-30 \%$. Each CM was titrated alone, and in the presence of the putative, synergistic regulator. The addition of known synergistic activities (e.g., PMA) served to unmask relatively weak CSAs. These concentration/responsiveness studies thus detected differential thresholds (either by examining broad ranges of $\mathrm{CM}$ alone, or by coculture) or inhibitors (by dilution). However, such studies could not exclude the presence of an inhibitory activity which had an identical response curve in both types of titration experiment. While improbable, such an event might reduce or mask lineage-specific or nonspecific activities. The concentrations routinely used were BICM $7.5 \%$, HLuCM 10\%, PHA-LCM 10\% (final concentrations).

Tumor-promoting phorbol diesters. Phorbol diesters (Sigma Chemical Co.) were prepared as stock solutions of $10^{-5} \mathrm{M}$ in dimethylsulfoxide and kept in the dark at $-20^{\circ} \mathrm{C}$ until used. Fresh stock solutions were prepared every $3 \mathrm{wk}$. Immediately before use, a working solution was made using culture medium as the diluent.

Human plasma samples. Plasma was taken from normal volunteers after obtaining informed consent. Plasma was anticoagulated with preservative-free heparin and immediately spun $(1,500 \mathrm{~g}$ for 20 $\mathrm{min}$ ) to produce platelet-poor plasma. This plasma was filter sterilized $(0.22 \mu \mathrm{m})$ and used immediately. Normal plasma, alone, did not stimulate colony development, but was screened for the optimal concentration supporting colony growth (30-40\%, final concentration).

Liquid suspension cultures. Bone marrow NALD cells were prepared as described above. After plastic adherence, the cells were subjected to nylon wool adherence to remove residual adherent cells and $B$ lymphocytes (20). Human marrow NALD-B cells or human erythroleukemia (HEL) cells (vide infra) were cultivated $\left(1-2 \times 10^{5} / \mathrm{ml}\right)$ in the presence of regulatory activities for 5 or $4 \mathrm{~d}$, respectively. Culture conditions were as described above.

\section{Human recombinant hematopoietic growth factors}

Recombinant human IL-3, GM-CSF, and IL-6 were the generous gift of Dr. Steven Clark, Genetics Institute, Cambridge, MA. The rh-IL-3 (lot 1039-86; sp act 0.5-1.0 $\times 10^{7} \mathrm{U} / \mathrm{mg}$ ) was Escherichia coli $\mathrm{ex}-$ pressed and 99\% pure. Human, rh-GM-CSF (lot CD19D2-701; sp act 1 $\times 10^{7} \mathrm{U} / \mathrm{mg}$ ) was $E$. coli expressed and $99 \%$ pure. Interleukin-6 was $E$. coli expressed, with sp act $5 \times 10^{6} \mathrm{U} / \mathrm{ml}$, and $\geq 95 \%$ pure. Recombinant IL-1 was purchased from Genzyme Corporation, Boston, MA.

\section{Solid-phase radioimmunoassay (SPRIA) for hematopoietic cell antigens}

In order to define, rapidly and precisely, factor-related changes in megakaryocyte development, we developed a SPRIA to detect megakaryocyte antigens (21). Briefly, the SPRIA used a filtration membrane as a solid support (Durapore, Millipore Corp., Bedford, MA) and antibody-bound ${ }^{125}$ I-staphylococcal protein A (SPA, ICN, Irvine, CA) as a detection system. Intact, cultivated bone marrow cells (nonadherent NALD-B or HEL cells), or other target cells, were loaded into microtiter wells, and all washes and incubations were performed in situ. Antibody binding was thus performed in a solid phase, i.e., antigens remained membrane-associated on intact cells which were affixed to the polyvinylidene membrane. Additionally, this procedure prevented cell loss due to repetitive centrifugation and wash steps. 
Calculations. Specific binding was defined as the total radioactivity (cpm) bound to antibody-labeled cells minus the radioactivity due to nonspecific binding of ${ }^{125}$ I-SPA to cells and secondary antibody. Nonspecific binding was $<15 \%$ of total binding for NALD-B cells or HEL cells $\left(5 \times 10^{4}\right.$ cell per well using antibody cocktail). Controls run to determine nonspecific ${ }^{125} \mathrm{I}$-SPA binding were $(a){ }^{125} \mathrm{I}$-SPA plus cells plus secondary antibody and $(b)$ cells plus ${ }^{125} \mathrm{I}$-SPA. Additional controls were run for each assay to determine the nonspecific binding of primary antibody, secondary antibody, or ${ }^{125}$ I-SPA to the polyvinylidene membrane. These controls detected alterations in membrane binding characteristics, molecular complexing of antibodies, etc. They were routinely $<1 \%$ of the nonspecific binding controls.

Antibody specificity. Three platelet/megakaryocyte-specific antibodies were used in these studies (21). These were: anti-Factor VIIlag (Dako Corp., Santa Barbara, CA), a monospecific heterologous antibody adsorbed three times with human WBCs, RBCs, and mouse liver powder; and two monoclonal antibodies-antiplatelet glycoprotein IIb/IIIa (a generous of Dr. Robb Todd, University of Michigan), and antiplatelet glycoprotein Ib (Dako Corp.).

Analysis of bone marrow cellular reactivity by immunocytochemistry (22) showed only mature and immature megakaryocyte positive for the peroxidase reaction (21). As well, antibody specificity in the RIA system was examined by using purified human PMNs, HL60 cells, and input NALD cells. None of these controls showed specific binding differing from zero.

The final RIA concentration of each antibody was as follows: antiFactor VIIIag 1:1000; anti-GP IIb/IIIa 1:30; and anti-Ib 1:20). In order to improve detection, a cocktail of these antibodies (each at optimal concentration) was used. All results reported were based on the signal detected using this antibody cocktail.

\section{HEL cell culture}

In order to precisely quantify factor responsiveness by RIA, human HEL cells were utilized as an additional target cell population. HEL cells are known to express many platelet/megakaryocyte-associated antigens $(21,23,24)$ and previous studies from this laboratory and those below have shown that the megakaryocyte antigenic content of these cells can be regulated (21). HEL cells were a generous gift of Dr. Thalia Papayannopoulou, University of Washington, Seattle, WA. HEL cells were cultivated in RPMI 1640 media (Gibco) containing $10 \%$ FCS, $1 \mathrm{mM}$ pyruvate, and $2 \mathrm{mM}$ glutamate. Incubation conditions were as those described above.

\section{Megakaryocyte morphology}

Megakaryocytes were identified using avidin-biotin immunoperoxidase conjugates (22) to label megakaryocyte colonies in situ or cytocentrifuge preparations of cells grown in suspension.

\section{Results}

Responsiveness of human megakaryocyte progenitor cells to various $C M$. Human megakaryocyte progenitor cells responded in vitro to either BICM or PHA-LCM. CM from the bladder carcinoma line 5637 supported limited megakaryocyte colony formation, stimulating $3.9 \pm 3$ megakaryocyte colonies $/ 10^{5}$ NALD cells cultured $(n=21$; Table I). These colonies consisted of 3-20 small, relatively immature megakaryocytes (data not shown). Equivalent numbers of megakaryocyte colonies (of similar size) were formed using PHA-LCM as a stimulus $(12.5 \pm 6, n=4)$. In addition to megakaryocyte-stimulating activity (Mk-CSA), both CM contained erythroid burst-promoting activity (BPA; Table I). Both CM also stimulated granulocyte colony formation, stimulating $\sim 100 \mathrm{CFU}-\mathrm{GM} /$ $10^{5}$ cells.

The low number of megakaryocyte progenitor cells responding to BlCM precluded the determination of Mk-CSA concentration-dependent responsiveness using this $\mathrm{CM}$ alone.
Table I. Responsiveness of Human Hematopoietic Progenitor Cells to Various Regulatory Activities

\begin{tabular}{|c|c|c|c|}
\hline Stimuli & CFC-Mk & CFC-GM & BFC-E $E^{\ddagger}$ \\
\hline & \multicolumn{3}{|c|}{ colonies per $10^{5}$ bone marrow cells* } \\
\hline Bladder CM & $4 \pm 3$ & $100 \pm 37$ & $\mathbf{0}$ \\
\hline PHA-LCM & $13 \pm 6$ & $94 \pm 44$ & $\mathbf{0}$ \\
\hline r-EPO & 0 & 0 & $\mathbf{0}$ \\
\hline PMA & 0 & 0 & 0 \\
\hline HLCM & 0 & $0^{\prime \prime}$ & 0 \\
\hline BlCM + HLuCM & $18 \pm 7^{\prime}$ & $125 \pm 20$ & NT \\
\hline $\mathrm{BICM}+10^{-8} \mathrm{M}$ PMA & $25 \pm 11^{\prime}$ & $106 \pm 17$ & NT \\
\hline $\mathrm{BlCM}+\mathrm{r}-\mathrm{EPO}^{\ddagger}$ & $10 \pm 7$ & NT & $12 \pm 6^{1}$ \\
\hline PHA-LCM + r-EPO ${ }^{\ddagger}$ & $15 \pm 2^{* *}$ & NT & $50 \pm 25^{9}$ \\
\hline r-IL-3 & $12 \pm 17$ & $72 \pm 42$ & NT \\
\hline r-IL-3 + $10^{-8}$ M PMA & $52 \pm 7^{1}$ & $83 \pm 17$ & NT \\
\hline r-GM-CSF & $11 \pm 7$ & $79 \pm 18$ & NT \\
\hline $\mathrm{r}-\mathrm{GM}-\mathrm{CSF}+10^{-8} \mathrm{M}$ PMA & $40 \pm 21^{\prime}$ & $61 \pm 25$ & NT \\
\hline Media/plasma & 0 & 0 & 0 \\
\hline$n$ & 21 & 25 & 5 \\
\hline
\end{tabular}

Human progenitor cells cultured in agar or methylcellulose as described in Methods. Values are mean \pm SD of three to four individual marrow samples cultured at three to five replicate cultures per condition. Abbreviations used in this table: CFC, colony-forming cell; BFC-E, burst forming cell erythrocyte; BICM, human bladder cell line 5637 CM (7.5\% final concentration); r-EPO, recombinant human erythropoietin ( $2 \mathrm{U} / \mathrm{ml}$, final concentration); PHA-LCM, phytohemagglutinin-stimulated human leukocyte-conditioned media (7.5-10\% vol/vol, final concentration); r-IL-3, recombinant human interleukin 3 tested at $1 \mathrm{ng} / \mathrm{ml}$; r-GM-CSF, recombinant granulocyte/macrophage CSF tested at $300 \mathrm{ng} / \mathrm{ml}$; NT, not tested.

* Target cells are NALD cells.

¥ Carried out in methylcellulose.

Media containing $30 \%$ human plasma as a control.

"High concentrations ( $20 \% \mathrm{vol} / \mathrm{vol})$ of HLuCM stimulate 7-10 granulocyte clusters $/ 10^{5}$ TNC.

'Significant observation ( $P \leq 0.05$, Student's $t$ test) contrast is vs. colony number stimulated by bladder CM alone; r-IL-3 or 4-GMCSF in the case of recombinant growth factors. Other contrasts (BlCM plus Epo and PHA-CM plus r-Epo) are not significant ( $P$ 20.05 ).

$* * n=3$.

However, such determinations were possible when cultures contained optimal concentrations of a second, synergistic activity (see below). Under such conditions, (i.e., holding the auxiliary activity at a constant concentration and titrating BlCM), megakaryocyte progenitor cells responded to BICM over a range of $1-20 \%$ (vol/vol; Fig. 1) with optimal colony formation occurring at 5.0-7.5\% (final concentration).

Synergistic interactions regulating in vitro megakaryocyte colony development. Observations of murine hematopoiesis have suggested the existence of synergistic activities that might augment human megakaryocyte development (8-11). In the megakaryocyte lineage, such activities are distinguished from CSAs in that they lack the solitary ability to support colony formation. However, when cocultured with a source of $\mathbf{M k -}$ CSA, these activities increase megakaryocyte colony detection, by increasing size, enzymatic content, cytoplasmic content, and DNA content of developing megakaryocytes (8-11, 25-28).

CM from A549 human lung lines contain an activity(ies) 


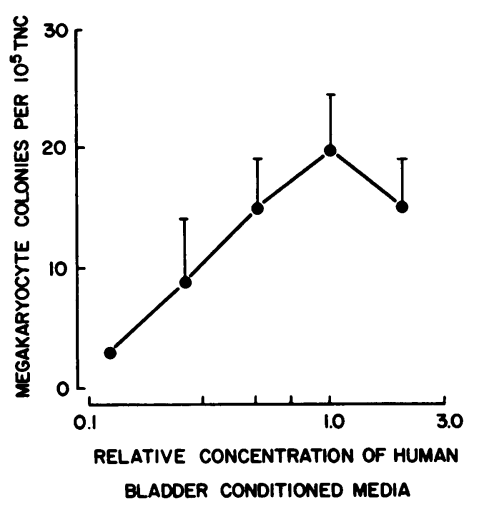

that augment(s) human megakaryocyte colony formation (Table I). Hence, $5637 \mathrm{CM}$, alone, stimulated $3.9 \pm 3$ colonies/ $10^{5}$ NALD cells cultured, whereas coculture of cells with BICM plus HLuCM ( 7.5 and $10 \%$, respectively) resulted in $17.6 \pm 7$ megakaryocyte colonies $/ 10^{5}$ cells $(P \leq 0.05$, Student's $t$ test).

The responsiveness of megakaryocyte progenitor cells to differing concentrations of this second, synergistic activity is presented in Fig. 2. In the presence of optimal concentrations of $\mathrm{BICM}(7.5 \%$, vol/vol), HLuCM significantly augmented megakaryocyte colony development over a range of $2.5-20 \%$ (vol/vol) with optimal, plateau colony formation occurring at 2.5\%. This amplification of megakaryocyte colony development was lineage specific inasmuch as no change was seen in granulocyte colony numbers at any concentration (Table I, titration data not shown).

Substitution of tumor-promoting phorbol diesters for biological activities regulating human megakaryocyte development. In a variety of cell systems, tumor-promoting phorbol diesters such as $4 \beta$-phorbol 12-myristate 13-acetate (PMA) are known to induce cellular differentiation (29-32). Examination of the effects of PMA on human bone marrow (NALD) cells shows that PMA, alone, lacks the ability to promote human megakaryocyte colony formation (i.e., it is not a Mk-CSA). However, tumor-promoting phorbols do substitute for HLuCM in augmenting megakaryocyte colony formation (Table I). In the presence of BlCM, $10^{-8} \mathrm{M}$ PMA stimulated $24.6 \pm 11$ megakaryocyte colonies $/ 10^{5}$ NALD cells $(P \leq 0.05$ Student's $t$ test, $n=21$ ). No effects of PMA (at the concentra-

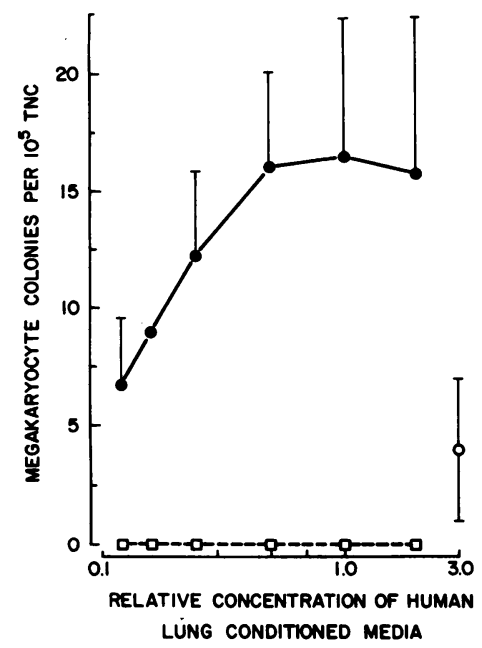

Figure 2. Responsiveness of human megakaryocyte progenitor cells to HLuCM. Cultures performed as in Methods. HLuCM responsiveness determined in the presence of BICM (7.5\% vol/vol). Relative concentration of 1.0 $=10 \%$ (final concentration, vol/vol). (๑) HLuCM from cell line A549. (ㅁ) HLuCM from cell line HEL 299. (O) Cells cultured in the presence of BICM only. Values are mean $\pm \mathrm{SD}(n=3)$. tions tested) were noted on granulocyte progenitor cell frequency. Thus, B1CM alone stimulated $100 \pm 37$ granulocyte colonies $/ 10^{5}$ cells whereas $\mathrm{BlCM}$ plus $10^{-8} \mathrm{M}$ PMA resulted in $107 \pm 17$ colonies. The responsiveness of human megakaryocyte progenitor cell to PMA was narrowly restricted (Fig. 3) with significant increases in colony numbers occurring at 6 $\times 10^{-9} \mathrm{M}$ and optimal effects seen at $10^{-8} \mathrm{M}\left(\mathrm{EC}_{50}=7\right.$ $\times 10^{-9} \mathrm{M}$ ).

Examination of the structure-activity relationship between various phorbol esters and megakaryocyte colony development indicates that the active tumor promoters PMA and phorbol 12,13-dibutyrate were capable of substituting for biological activities that augment megakaryocyte colony formation (Table II). In contrast, the non-tumor-promoting parent alcohol (phorbol) as well as dimethylsulfoxide (the vehicle) did not enhance megakaryocyte colony development.

Role of recombinant human hematopoietic growth factors in megakaryocyte development. In order to identify hematopoietic factors capable of regulating megakaryocyte development, we examined recombinant human IL-3, IL-6, IL-1, erythropoietin, and GM-CSF for their solitary ability to support megakaryocyte colony formation, or for their capacity to augment CSF-driven colony formation. IL-3 alone supported colony formation over a range of $100-1,000 \mathrm{pg} / \mathrm{ml}$ (Fig. 4). Nonetheless, addition of PMA to IL-3-containing cultures significantly augmented colony formation at all concentrations of IL-3 tested. GM-CSF stimulated equivalent megakaryocyte colony formation but required an approximate 100 -fold increase in concentration (Table I). Synergistic interactions also occurred between GM-CSF and phorbol diesters. Thus, at 300 ng of GM-CSF, 4 \pm 1 megakaryocyte colonies per $10^{5}$ TNC were observed, whereas addition of $10^{-8} \mathrm{M}$ PMA yielded $48 \pm 23$ colonies (mean $\pm S D ; n=3$ ). Finally, recombinant erythropoietin, IL-1 or IL-6 did not show solitary colony stimulating or synergistic interactions when tested (0-100 and 0.1-10.0 U/ml, respectively) against IL-3 in a range of $0.1-1,000 \mathrm{ng} / \mathrm{ml}$ ( $n=4$; data not shown).

Morphologic analysis of megakaryocytes grown in suspension phase cultures. A substantial increase in size and colony cellularity (3-100 megakaryocytes) occurred when progenitor cells were grown under optimal conditions (data not shown).

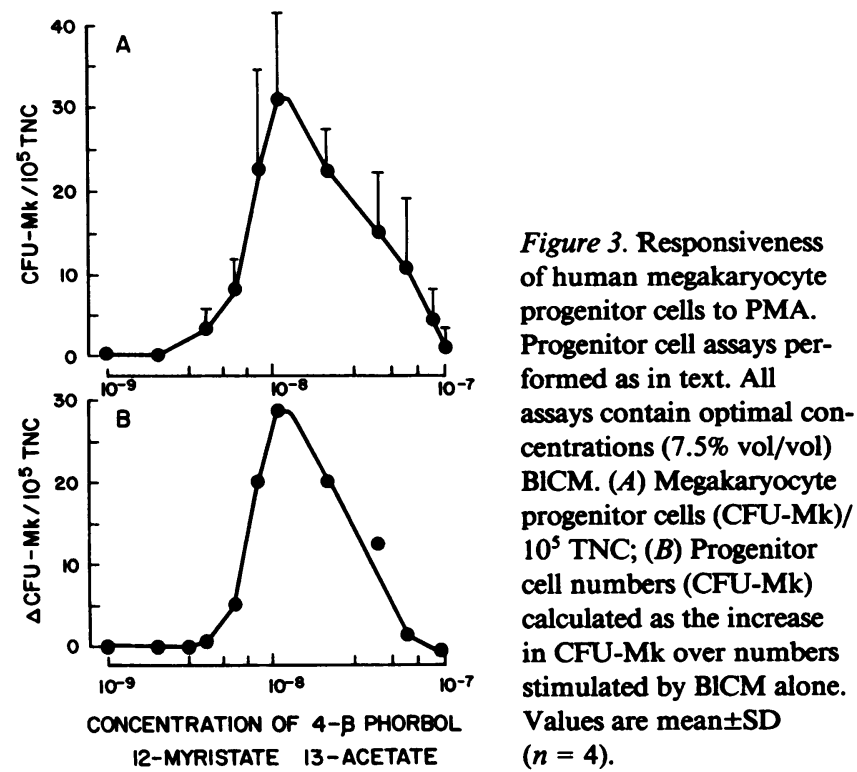


Table II. Structure-Activity Relationship between Various Phorbol Esters and Megakaryocte-potentiating Ability

$\begin{array}{lc}\text { Phorbol ester } & \begin{array}{c}\text { Relative synergistic } \\ \text { activity }\end{array} \\ & \% \\ \text { PMA } & 100 \\ \text { PDBu } & 98 \\ \text { Phorbol } & 0 \\ \text { DMSO (vehicle) } & 0\end{array}$

For comparison, the maximum synergistic activity of $10^{-8} \mathrm{M}$ PMA, when cocultured with optimal amounts of BLCM (7.5\% final concentration) is expressed as 100 . All phorbol esters were tested at $10^{-8}$ $M$. Values are means of four individual marrow samples each cultured at three to five replicate culture/experiment/condition. Optimal value of BLCM + PMA represents $24.6 \pm 11.3 \mathrm{CFU}-\mathrm{Mk} / 10^{5}$ TNC. Abbreviation: PDBu, phorbol 12,13-dibutyrate.

Morphologic analysis of megakaryocytes grown in suspension cultures shows great differences in megakaryocyte phenotype (Table III). When cultured for $5 \mathrm{~d}$ in the presence of Mk CSA, megakaryocytes had a high nucleus/cytoplasm ratio, were of small size, and contained low amounts of megakaryocytic antigens (as visualized by immunoperoxidase). The addition of PMA or HLuCM to BICM containing cultures resulted in a reversal of the nucleus/cytoplasm ratio (i.e., an increased cytoplasmic content), a larger size, and a subjective increase in antigenic content/megakaryocyte. Interestingly, the addition of HLUCM or PMA to BICM-containing cultures increased the number of antigen-positive cells in these cultures. HLuCM or PMA also increased the maturity of developing megakaryocytes in a concentration-dependent manner (Table III).

Factor-related increases in megakaryocyte antigens. The use of a SPRIA has the advantage of being an accessory cellfree system which allows a sensitive, rapid, and independent confirmation of regulatory differences in in vitro megakaryocyte development (21).

Two major sources of variability exist in the bioassay phase (i.e., suspension cultures) of the SPRIA analysis. One is the presence of a detectable regulatory activity in normal plasma (vide infra). The other source of variability is the inherent biological differences in human donors. The combined plasma/marrow variability results in a large interassay variability index. However, within each assay distinct differences in factor-stimulated SPA binding are seen. For purposes of

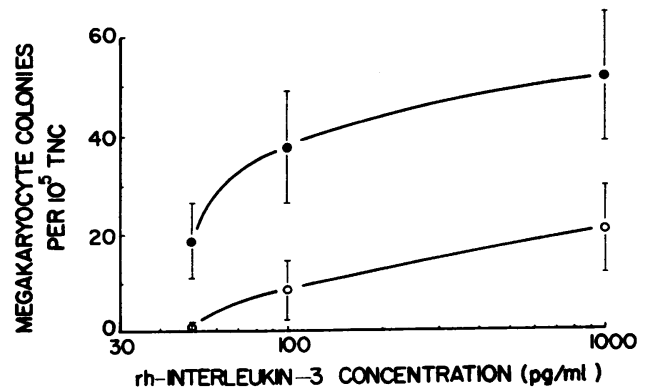

Figure 4. Synergistic interactions between rh-IL-3 and phorbol diesters (PMA). Megakaryocytes cultured as in Methods. (0) rh-IL-3 alone at indicated concentrations; (๑) rh-IL-3 plus $10^{-8}$ M PMA. Results are mean $\pm \operatorname{SEM}(n=4)$ based on three replicate cultures per point.
Table III. Morphologic Changes in Suspension-Phase Megakaryocytopoiesis

\begin{tabular}{|c|c|c|c|}
\hline \multirow[b]{2}{*}{ Stimuli } & \multirow[b]{2}{*}{$\mathrm{Mk} / 10^{5} \mathrm{TNC}$} & \multicolumn{2}{|c|}{ Mk classification } \\
\hline & & Immature & Mature \\
\hline & & \multicolumn{2}{|c|}{$\%$} \\
\hline Bladder CM & $360 \pm 82$ & 100 & 0 \\
\hline $\mathrm{BlCM}+10^{-8} \mathrm{M}$ PMA & $3151 \pm 56$ & 53 & 47 \\
\hline \multicolumn{4}{|l|}{$\mathrm{BlCM}+\mathrm{HLuCM}$} \\
\hline $10.0 \%^{\ddagger}$ & 233 & 100 & 0 \\
\hline $5.0 \%$ & 466 & 71 & 39 \\
\hline $2.5 \%$ & 750 & 73 & 27 \\
\hline $1.0 \%$ & 366 & 90 & 10 \\
\hline $0.1 \%$ & 167 & 100 & 0 \\
\hline
\end{tabular}

Megakaryocytes grown in suspension phase cultures as in Methods. Quantitative values are mean $\pm \mathrm{SD}(n=3)$ except for $\mathrm{BICM}$ + HLuCM titration which are five separate cultures from an individual marrow donor. Abbreviations as in Table I.

* Megakaryocytes identified by immunoperoxidase and classified as: immature cells $\leq 18 \mu \mathrm{m}$ diam, high nucleus/cytoplasm content (see references 22-24) and low amounts of antigen (trace-1+), and mature cells being $\geq 20 \mu \mathrm{m}$ diam, low nucleus/cytoplasm ratio and large amounts of antigen $(++$ to ++++$)$, for classification $\sim 100$ megakaryocytes (mature and immature) were counted per condition/ donor.

¥ Percentages are amount (vol/vol) of HLuCM added to a constant concentration of $\mathrm{BICM}(7.5 \%$, vol/vol).

further hypothesis testing, ${ }^{125} \mathrm{I}-\mathrm{SPA}$ binding is normalized and expressed as the fold-increase over BSA controls. The presence of BICM slightly increased SPA binding in these cultures. A significant $(P \leq 0.05)$, synergistic increase in megakaryocytic antigens was seen when NALD cells were cocultured for $5 \mathrm{~d}$ with BICM and either $10^{-8}$ M PMA or HLuCM. BICM or HLuCM, alone, stimulated a three- to sevenfold increase in specific ${ }^{125}$ I-SPA binding, again with a large degree of variability (data not shown).

A notable difference between the semisolid and suspension culture systems is the detection of a regulatory activity in the plasma of normal individuals (Fig. $5 \mathrm{~A}$ ). Using BSA media as a control for the amount of protein in these cultures, the specific binding of ${ }^{125} \mathrm{I}$-SPA to megakaryocytes was not significantly different from input levels $\left(23.7 \mathrm{pg} / 10^{5} \mathrm{TNC}\right.$ input vs. 29.3 for 5-d BSA-conditioned cells). In contrast, the addition of $30 \%$ human platelet-poor plasma resulted in a significant increase ( $P \leq 0.05$ Student's $t$ test, two-tailed) in the total amount of megakaryocytic antigens. Plasma was prepared, fresh, for each assay inasmuch as we (and others; see references 13 and 17) found human sera to be inhibitory in colony culture systems (not shown). RIA analysis of 10 donor plasmas indicated seven stimulatory, two inhibitory, and one neutral for this megakaryocytic activity (also not shown). While significant increases in factor-driven antigenic content occurred within $5 \mathrm{~d}$, synergistic interactions were not noted until days 10-12 (Fig. 5 B). Again synergistic increases were observed with the addition of PMA or HLuCM.

These regulatory activities also induced increased antigenic expression HEL cells (Fig. 6). As reported elsewhere (21, 23, 24), these cells constitutively expressed megakaryocytic antigens. Stimulation with $10^{-8}$ to $10^{-9} \mathrm{M}$ PMA resulted in a significant $(P \leq 0.05)$ increase in antigenic expression. Simi- 

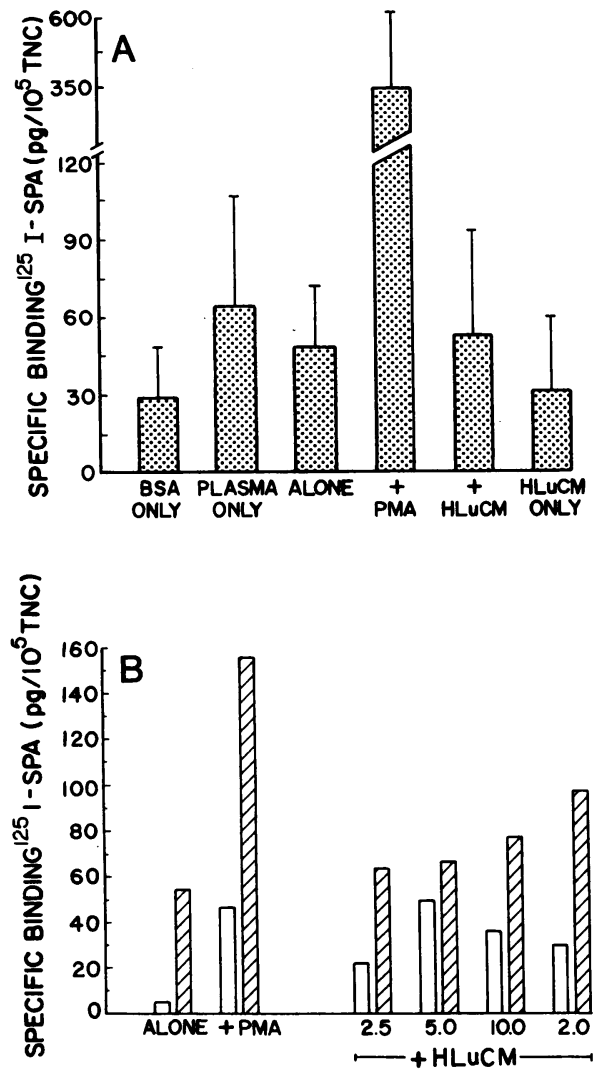

Mk-CSA CONTAINING CULTURES

Figure 5. Factor regulation of human bone marrow megakaryocyte antigenic content. SPRIA performed as in Methods. $(A)$ Stimulation of antigenic content after $5 \mathrm{~d}$ of culture. Values are mean $\pm \mathrm{SD}(n$ $=11$, except for BSA which is 9). (B) Comparison of day 5 (open bars) versus day 12 (hatched bars) antigenic content. Numbers below HLuCM bars are the percent concentration of CM (vol/vol). The source of Mk-CSA is BICM $(7.5 \% \mathrm{vol} / \mathrm{vol})$. Values are means of triplicate determinations from a single representative experiment of three separate marrow donors.

larly, stimulation with HLuCM while not as powerful as PMA resulted in a doubling of antigenic content.

\section{Discussion}

The regulation of human megakaryocyte colony development requires the presence of at least two factors that promote both proliferation and differentiation of this progenitor cell. Media conditioned by human bladder carcinoma cells (line 5637), human peripheral blood leukocytes (PHA-LCM), as well as rh-IL-3 or rh-GM-CSF support limited megakaryocyte colony development. Megakaryocyte colony growth is greatly augmented by the addition of CM from neoplastic lung cell lines or tumor-promoting phorbol diesters, suggesting the existence of dual or multiple levels of regulation in this process. This synergistic activity lacks the solitary ability to promote progenitor cell proliferation. Instead, it amplifies megakaryocytopoiesis by increasing the number and size of developing megakaryocytes. Importantly, this regulator also increases megakaryocyte antigenic determinants as detected by immunoperoxidase labeling and SPRIA.

The optimal formation of human megakaryocyte colonies

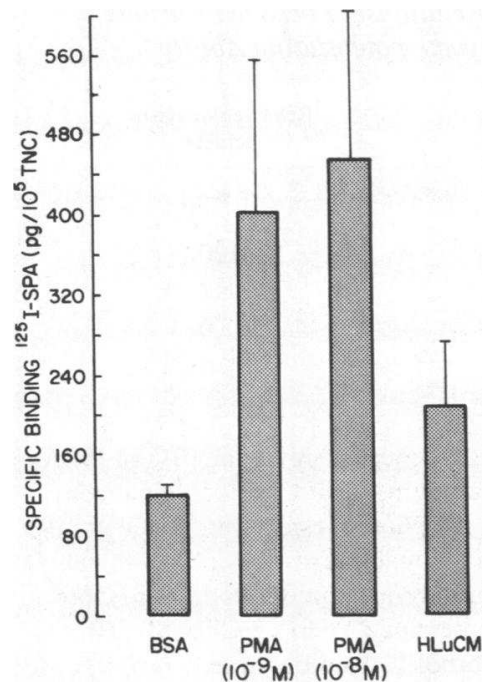

Figure 6. Responsiveness of HEL Cells to phorbol diesters and HLuCM. Cultures and SPRIA performed as in Methods. Values are mean $\pm \mathrm{SD}(n$ $=9$ except for HLuCM, which is 6).

requires synergistic interactions between two classes of regulatory activities: one supporting proliferation and differentiation of the progenitor cells (Mk-CSA) and the second enhancing megakaryocyte colony development but lacking the solitary ability to drive colony formation. Mk-CSA is found in BlCM and PHA-LCM. The presence of megakaryocyte regulatory activities in PHA-LCM is well documented using both plasma clot and methylcellulose cultures $(12,13,17)$. Gabrilove et al. (33) report the purification of a multilineage regulator from 5637 bladder carcinoma cells that stimulates both granulocytic and erythrocytic colonies. Thus, 5637 conditioned media (BICM) contains at least one regulator with multilineage effects. Alternatively, these bladder carcinoma cells may produce other factors affecting megakaryocytes, one or more of which is present in limiting (suboptimal) concentrations.

To establish which factors in these complex media might control megakaryocyte proliferation, we examined the actions of rh-IL-3 and GM-CSF. Both factors support proliferation of megakaryocyte progenitor cells, but limited maturation. These observations extend and confirm previous reports of murine megakaryocyte colony formation in the presence of recombinant growth factors (7). While colony numbers are higher in the presence of recombinant factors than in $\mathrm{CM}$, development is suboptimal unless another regulatory factor is present (e.g., PMA). Phorbol diesters increase colony number and size presumably by increasing cell/colony detection due to increased cellular maturation (vide infra). Nonetheless, the use of recombinant growth factors and PMA as a substitute for the biological co-regulator (see References 10 and 11 and Results) allows unambiguous confirmation of the need for two factors in megakaryocyte proliferation and differentiation.

Our data show that BlCM, hr-IL-3, or hr-GM-CSF stimulates a low number of small (immature) megakaryocytes. The addition of a synergistic co-regulator present in HLuCM (or its substitute PMA) to these cultures increases the number, size, cytoplasmic content, antigenic content, and maturity of developing megakaryocytes. The increase in the number of developing megakaryocytes suggests that this activity either augments proliferation (without supporting colony formation) or, alternatively, regulates the expression of megakaryocyte antigens making previously unrecognizable cells antigen-positive. This latter hypothesis is substantiated by the observation of increased numbers of antigen-positive megakaryocytes in sus- 
pension cultures. The actions of this auxiliary regulatory activity are lineage specific inasmuch as neither HLuCM (or its substitute PMA) support or affect granulocytic colony formation. Further, this activity is not a function of erythropoietin, IL-1, or IL-6 in that these recombinant proteins fail to support megakaryocyte colonies either alone or in conjunction with sources of IL-3.

The detection of increased megakaryocyte antigenic determinants by SPRIA independently substantiates our observations of megakaryocyte development in human bone marrow cultures. In a suspension culture system, HLuCM or PMA stimulation increases antigen levels over that seen with BICM alone. Interestingly, comparison of plasma-containing cultures and cultures containing and an equivalent amount of BSA indicates that normal human plasma contains low, but detectable, levels of this synergistic regulator. The SPRIA detects total megakaryocyte antigenic content (per $10^{5} \mathrm{TNC}$ ) which is a function of both the number and size of the megakaryocytes. Thus, the assay does not distinguish between proliferation or differentiation events. However, the morphologic and quantitative analysis of cultured megakaryocytes described above makes possible such distinctions.

It is interesting that the sensitivity of the SPRIA is such that differences in factor-driven increases in antigenic regulation are observed within $5 \mathrm{~d}$ of incubation (vs. 14-16 d for colony detection). Antigenic content continues to increase in suspension until days 10-12 at which point synergistic growth factor interactions are observed. These differences in regulation are presumably due to technical (liquid vs. semisolid culture) or biological considerations which are currently under investigation.

Both PMA and the biological source of the synergistic megakaryocyte regulator (HLuCM) also stimulate megakaryocyte antigen expression in HEL cells. These observations confirm and extend the work of Papayannopoulou et al. (23) and others (24) showing the presence and induction of megakaryocyte antigens in HEL cells. Importantly, we show that a biological activity capable of influencing the development of native human megakaryocytes also effects megakaryocytic expression in HEL cells. This implies the presence of a receptor for such an activity(ies) and suggests the usefulness of this cell line as a model for studies on the mechanisms of megakaryocyte development.

The actual target cell(s) for the actions of IL-3, GM-CSF, or the secondary synergistic activity are unknown. Two potential megakaryocyte target cell populations exist in suspension cultures: megakaryocyte progenitor cells, and immature megakaryocytes. Such immature cells are observed in immunocytochemically labeled NALD cell populations (34, and $M$. Long, unpublished observations). These studies utilize partially fractionated (NALD) cells at limiting dilutions $(50,000 /$ $\mathrm{ml}$ ), suggesting that accessory cells play a minor, if any, role in factor responsiveness. However, the use of $C M$ rather than purified activities, and accessory cell-depleted (NALD-B) rather than purified cells, makes direct assessment of the role of these factors and their targets difficult. Thus, it cannot be concluded that accessory cells are not involved in these studies, or that more than one synergistic regulator is present. Given these limitations, further characterization of the role of the co-regulator and its target cells must await purification of the synergistic regulatory factor and the use of purified target cell populations.

Two-tiered regulation of human megakaryocyte develop- ment was first described by Harker and Finch who proposed that separate activities regulate expansion versus the size of developing megakaryocytes (5). Hoffman et al. (6) observed that purified Mk-CSA acts at the level of megakaryocyte progenitor cells and suggested that activities such as thrombopoietin regulate more distal events. Consistent with this hypothesis, Gewirtz et al. (35) failed to detect Mk-CSA in patients with reactive thrombocytosis, and proposed the presence of a second regulator in these patients.

Certain correlations exist between in vivo and in vitro regulation of megakaryocyte development. Studies of murine thrombopoiesis in vivo show rapid changes in megakaryocyte development after perturbations of platelet concentration. Early after thrombocytopenia, megakaryocytes increase in size, cytoplasmic content, and maturity (36-39), and thrombopoietin activity is detectable in thrombocytopenic plasma/ sera $(40,41)$. However, little change is seen in megakaryocyte number unless stimulation is prolonged $(42,43)$. These observations imply two phases of in vivo regulation occur: an early thrombopoietin response affecting megakaryocyte/platelet development, and a later phase affecting megakaryocyte number (proliferation). In vitro, Hoffman and co-workers (6) show that purified Mk-CSA regulates the proliferation of megakaryocyte progenitor cells, stimulating the formation of colonies of small, immature megakaryocytes. We report a cofactor synergistic to Mk-CSA which regulates megakaryocyte size, cytoplasmic content, antigenic expression, and maturity. Thus, the in vitro actions of this synergistic activity mimic some of the known in vivo effects of thrombopoietin. Whether these regulators (i.e., thrombopoietin and the in vitro synergistic activity) are different molecules, or members of a family of regulators, must await the purification of both factors.

We conclude that optimal human megakaryocyte development requires the presence of two synergistic regulatory activities. Regulation of megakaryocyte proliferation is an activity of IL-3 or GM-CSF, either of which is obligate for colony formation and stimulates limited proliferation and differentiation of megakaryocytes. Full colony formation requires a second activity that, lacking CSA, increases megakaryocyte colony numbers and, in suspension cultures, increases the number, size, cytoplasmic content, antigenic content, and maturity of developing megakaryocytes thus mimicking some of the known actions of thrombopoietin.

\section{Acknowledgments}

The authors are indebted to Dr. Steven Clark for recombinant proteins, Dr. Hans Messner for his generous efforts in showing us the methylcellulose culture system, Nazir Jamal for teaching and advice concerning this system, and Terry Oliver for careful preparation of this manuscript.

This study was supported in part by grants HL-31568 from the National Institutes of Health and 83-1169 from the American Heart Association. Dr. Long is a Scholar of the Leukemia Society of America.

\section{References}

1. Gregory, C. J., and A. C. Eaves. 1977. Human marrow cells capable of erythropoietic differentiation in vitro: definition of three erythroid colony responses. Blood. 49:855-864.

2. Iscove, N. N. 1977. The role of erythropoietin in regulation of population size and cell cycling of early and late erythroid precursors in mouse bone marrow. Cell Tissue Kinet. 10:323-334.

3. Bradley, T. R., and G. S. Hodgson. 1979. Detection of primitive 
macrophage progenitor cells in mouse bone marrow. Blood. 54:14461458.

4. Bartelmez, S. H., and E. R. Stanley. 1985. Synergism between hemopoietic growth factors (HGFs) detected by their effects on cells bearing receptors for a lineage specific HGF: assay of hemopoietin-1. $J$. Cell. Physiol. 122:370-378.

5. Harker, L. A., and C. N. Finch. 1969. Thrombokinetics in man. J. Clin. Invest. 48:963-974.

6. Hoffman, R., H. H. Yang, E. Bruno, and J. E. Straneva. 1982. Purification and partial characterization of a megakaryocyte colonystimulating factor from human plasma. J. Clin. Invest. 75:1174-1182.

7. Quesenberry, P. J., J. N. Ihle, and E. McGrath. 1985. The effect of interleukin-3 and GM-CSA-2 on megakaryocyte and myeloid clonal colony formation. Blood. 65:214-217.

8. Williams, N., R. R. Eger, H. M. Jackson, and D. J. Nelson. 1982. Two-factor requirement for murine megakaryocyte colony formation. J. Cell. Physiol. 110:101-104.

9. Williams, N., H. Jackson, P. Ralph, and I. Nakoinz. 1981. Cell interactions influencing murine marrow megakaryocytes: nature of the potentiator cell in bone marrow. Blood. 57:157-163.

10. Long, M. W., J. E. Smolen, P. Szczepanski, and L. A. Boxer. 1984. Role of phorbol diesters in in vitro murine megakaryocyte colony formation. J. Clin. Invest. 74:1686-1692.

11. Long, M. W., L. L. Gragowski, C. H. Heffner, and L. A. Boxer. 1985. Phorbol diesters stimulate the development of an early murine progenitor cell. J. Clin. Invest. 76:431-438.

12. Messner, H. A., N. Jamal, and C. Izaguirre. 1982. The growth of large megakaryocyte colonies from human bone marrow. J. Cell. Physiol. 1:45-51.

13. Kimura, H., S. A. Burstein, D. Thorning, J. S. Powell, L. A. Harker, P. J. Fialkow, and J. W. Adamson. 1984. Human megakaryocytic progenitors (CFU-M) assayed in methylcellulose: physical characteristics and requirements for growth. J. Cell. Physiol. 118:87-96.

14. Mazur, E. M., R. Hoffman, and E. Bruno. 1981. Regulation of human megakaryocytopoiesis: an in vitro analysis. J. Clin. Invest. 68:733-741.

15. Hoffman, R., E. Mazur, E. Bruno, and V. Floyd. 1981. Assay of an activity in the serum of patients with disorders of thrombopoiesis that stimulates formation of megakaryocytic colonies. N. Engl. J. Med. 305:533-538.

16. Mazur, E. M., P. DeAlarcon, K. South, and L. Miceli. 1984 Human serum megakaryocyte colony stimulating activity increases in response to intensive cytotoxic chemotherapy. Exp. Hematol. 12:624-628.

17. Solberg, L. A., Jr., N. Jamal, and J. A. Messner. 1985. Characterization of human megakaryocytic formation in human plasma. $J$. Cell. Physiol. 124:67-74.

18. Williams, N., H. M. Jackson, R. R. Eger, and M. W. Long. 1981. The separate roles factors in murine megakaryocyte colony formation. In Megakaryocyte Biology and Precursors: Cloning and Cellular Properties. B. L. Evatt, R. F. Levine, and N. Williams, editors. Elsevier, Amsterdam. 69-75.

19. Long, M. W., and N. Williams. 1982. Differences in the regulation of megakaryocytopoiesis in the murine bone marrow and spleen. Leukemia Res. 6:721-728.

20. Niederhuber, J. E., and P. Allen. 1980. The role of Ia gene products in macrophage induction of antibody response. II. Restriction of levels of $\mathrm{T}$ cell in the recognition of I-I macrophage detection. $J$. Exp. Med. 151:1103-1113.

21. Long, M. W., and C. H. Heffner. 1988. Detection of human megakaryocyte antigens by solid-phase radioimmunoassay. Exp. $\mathrm{He}$ matol. 16:62-70.

22. Hsu, S. M., L. Raine, and H. Fanger. 1981. The use of avidinbiotin-peroxide complex $(\mathrm{ABC})$ in immunoperoxidase technique: a comparison between $\mathrm{ABC}$ and unlabeled antibody (PAP) procedures. J. Histochem. Cytochem. 29:577-580.

23. Papayannopoulou, T., T. Yokochi, B. Nakamoto, and P. Mar- tin. 1983. The surface antigen expression of HEL cells. In Globin Gene Expression and Hematopoietic Cell Differentiation. G. Stamatoyarrapoulous and A. Neinhuis, editors. Alan R. Liss, Inc., New York. 277-292.

24. Tabilio, A., J. P. Rosa, N. Kieffer, A. T. Nurden, M. C. Del Canizo, J. Breton-Gorus, and W. V. Ainchanker. 1984. Expression of platelet membrane glycoproteins and granule proteins by a human erythroleukemia cell line (HEL). EMBO (Eur. Mol. Biol. Organ.) J. 3:453-459.

25. Long, M. W., and N. Williams. 1981. Immature megakaryocytes in the mouse: morphology and quantification by acetylcholinesterase staining. Blood. 58:1032-1039.

26. Long, M. W., and N. Williams. 1982. Immature megakaryocytes in the mouse: characteristics, cell cycle status and in vitro responsiveness to thrombopoietic stimulatory factor. Blood. 59:569-575.

27. Long, M. W., N. Williams, and T. P. McDonald. 1982. Immature megakaryocytes in the mouse: in vitro relationship to megakaryocyte cells mature megakaryocytes. J. Cell. Physiol. 112:339-344.

28. Williams, N., and H. Jackson. 1982. Kinetic analysis of megakaryocyte numbers and ploidy levels in developing colonies from mouse bone marrow cells. Cell Tissue Kinet. 15:483-494.

29. Ebeling, J. G., G. R. Vandenbark, L. J. Kuhn, B. R. Ganong R. M. Bell, and J. E. Niedel. 1985. Diacyglycerols mimic phorbol diester induction of leukemic cell differentiation. Proc. Natl. Acad. Sci. USA. 82:815-819.

30. Fibach, E., P. A. Marks, and R. A. Rifkin. 1980. Tumor promotors enhance myeloid and erythroid colony formation by normal mouse hematopoietic cells. Proc. Natl. Acad. Sci. USA. 77:4152-4155.

31. Nishizuka, Y. 1984. The role of protein kinase $C$ in cell surface signal transduction and tumor promotion. Nature (Lond.). 308:693698.

32. Rozengurt, E., M. Rodriguez-Pena, and K. A. Smith. 1983. Phorbol esters, phospholipase $\mathrm{C}$, and growth factors rapidly stimulate the phosphorylation of a $M_{\mathrm{r}} 80,000$ protein in intact quiescent $3 \mathrm{~T} 3$ cells. Proc. Natl. Acad. Sci. USA. 80:7244-7248.

33. Gabrilove, J. C., K. Welte, P. Harris, E. Platzer, L. Lu, E. Levi, R. Mettelsmann, and M. A. S. Moore. 1985. Pluripoietin-alpha: A second human hematopoietic colony stimulating factor produced by bladder carcinoma cell line 5637. Proc. Natl. Acad. Sci. USA. 83:247248.

34. Rabellino, E. R., R. B. Levene, L. L. L. Leung, and R. L. Nachman. 1981. Human Megakaryocytes. II. Expression of platelet proteins in early marrow megakaryocytes. J. Exp. Med. 154:88-100.

35. Gewritz, A. M., E. Bruno, J. Elwell, and R. Hoffman. 1983. In vitro studies of megakaryocytopoiesis in thrombocytotic disorders of man. Blood. 61:384-389.

36. Odell, T. T., and C. Shelton. 1979. Increasing stimulation of megakaryocytopoiesis with decreasing platelet count. Proc. Soc. Exp. Biol. Med. 161:531-533.

37. Odell, T. T., J. R. Murphy, and C. W. Jackson. 1976. Stimulation of megakaryocytopoiesis by acute thrombocytopenia in rats. Blood. 48:765-775.

38. Odell, T. T., C. W. Jackson, T. J. Friday, and D. E. Charsha. 1969. Effects of thrombocytopenia on megakaryocytopoiesis. $B r . J$. Haematol. 17:91-101.

39. Ebbe, S. 1976. Biology of megakaryocytes. Prog. Hemostasis Thromb. 3:211-229.

40. Harker, L. A. 1970. Regulation of thrombopoiesis. Am. J. Physiol. 218:1376-1380.

41. Levin, J., and B. L. Evatt. 1979. Humoral regulation of thrombopoiesis. Blood Cells (Berl.). 5:105-121.

42. Harker, L. A. 1968. Kinetics of thrombopoiesis. J. Clin. Invest. 47:458-465.

43. Odell, T. T., C. W. Jackson, T. J. Friday, and D. E. Charsha. 1969. Effects of thrombocytopenia on megakaryocytopoiesis. Br. J. Haematol. 17:91-101. 